\title{
Life Prediction Method of Power Battery for New Energy Vehicle
}

\author{
Guanqiang Ruan,Jinliang Cao,Xing Hu* \\ Mechanical Engineering School, Shanghai Dian Ji University, Shang Hai, China.
}

\begin{abstract}
Battery life is a key factor restricting the development of new energy vehicle manufacturing industry.Taking a $\mathrm{LiMn}_{2} \mathrm{O}_{4}$ power battery as an example, the life prediction method of power battery based on performance degradation data was studied. It is found that the capacity of the battery basically conforms to the decline trajectory of the power function, and the pseudo failure life of the battery is extrapolated by using the decline model. The distribution of pseudo failure life is studied, and the results are consistent with the fitting effect of Weibull distribution. Finally, the reliability of the battery is evaluated based on Weibull life distribution. This paper analyzes the influence of the actual cycle data on the prediction accuracy. The test results show that the life model and reliability evaluation method have high accuracy, and solve the problems of long life evaluation cycle and high cost of power battery.
\end{abstract}

Keywords: Lithium ion power battery, performance degradation data, battery life prediction, life assessment.

\section{Introduction}

The electric vehicle or hybrid vehicle made by the combination of lithium-ion battery and automobile power can effectively save energy and reduce the air pollution caused by automobile exhaust, which meets the needs of green travel and low-carbon life [1-2]. China has gradually formed a mature power lithium-ion battery market.

Although lithium-ion battery has a wide range of application space, there are still some problems in the actual production and use process, which affect and limit the development of lithium-ion battery and need our attention. How to improve the technical level of lithium-ion battery has almost become a key factor affecting the development of electric vehicles.

In the electric vehicle industry, the battery cost accounts for $40 \%$ of the total cost of the automobile, and the proportion is higher. The life, safety and cost of lithium-ion battery directly affect the expansion of new energy vehicles. How to improve the performance and reduce the cost of lithium-ion battery has become the research direction in the future [3]. However, the internal mechanism of lithium-ion battery is very complex, which is a nonlinear dynamic electrochemical system. With the increase of charge discharge cycles, the performance state of lithium-ion battery will degrade slowly. When the performance state degenerates to a certain extent, it is considered that the life of lithium-ion battery is terminated. Therefore, in order to improve the utilization rate, control the battery capacity decay and prolong the service life, it is necessary to study the decay mechanism of lithium-ion battery and establish a recession model to predict the remaining life [4-5]. This is not only of great significance to the development of electric vehicles, but also has a guiding role in other battery technologies.

\section{Study on characteristics of lithium ion battery}

\subsection{Structure of lithium ion battery}

There are two kinds of lithium ion batteries: cylindrical and square, which are generally composed of five parts, namely, positive electrode, diaphragm, negative electrode, electrolyte and shell. The specific structure is as follows: (1) Positive electrode: The active material of the positive electrode of lithium ion battery is generally lithium manganate, lithium cobaltate or nickel cobalt lithium manganate. When charging, lithium ions are extracted 
from the interlayer of the positive electrode, and the positive electrode emits electrons. (2) Diaphragm: It is a kind of polymer film with microporous structure. The function of this film is to separate the positive and negative electrodes of the battery and prevent short circuit caused by contact between the two electrodes. In addition, electrons cannot pass through the diaphragm, but lithium ions can pass freely. Generally a polyolefin porous membrane. (3) Negative electrode: The negative active material of lithium ion battery is graphite or carbon. When discharging, lithium ions are pumped out from the negative electrode and returned to the positive electrode layer. (4) Electrolyte: The ionic conductor that plays a conductive role between the positive and negative electrodes is electrolyte. When the charging voltage is above $4 \mathrm{~V}$, because of the high voltage, instead of using aqueous solution as electrolyte, an electrolyte that can withstand high pressure and the electrolyte is dissolved in a stable organic solvent is used. Generally, it is a carbonate solvent in which lithium hexafluorophosphate is dissolved. (5) Shell: also called battery container, which has a battery cap, from which the positive and negative electrodes of the battery can be led out [6-7].

\subsection{Working principle of lithium ion battery}

Taking the working principle of lithium iron phosphate battery as an example, in the charging process of $\mathrm{LiFePO}_{4}$ battery, lithium iron phosphate in the positive electrode loses electrons, lithium ion $\mathrm{Li}^{+}$migrates out, and lithium ion migrates to the negative electrode due to the traction of electric field force. On the contrary, during the discharge process of $\mathrm{LiFePO}_{4}$ battery, the lithium ion $\mathrm{Li}^{+}$in the negative electrode migrates from the interlayer of graphite negative electrode and migrates to the positive electrode through electrolyte. Lithium ions migrate back and forth during each charge and discharge process, as follows:

When the battery is charged, $\mathrm{Li}^{+}$escapes from the lithium iron phosphate crystal, enters the electrolyte, moves to the graphite crystal surface of the negative electrode under the traction of the electric field force, and is embedded in it. At the same time, electrons will flow to the aluminum foil collector of the positive electrode through the conductor, then to the copper foil collector of the negative electrode, and then flow to the graphite negative electrode through the conductor, finally making the negative electrode charge balance.

When the battery is discharged, $\mathrm{Li}^{+}$escapes from the graphite crystal and enters the electrolyte, migrates to the surface of lithium iron phosphate crystal, and then re-embeds into the crystal lattice of lithium iron phosphate. At this time, electrons flow to the copper foil collector of the negative electrode through the conductor, then to the aluminum foil current collector of the positive electrode of the battery, and then flow to the lithium iron phosphate positive electrode through the conductor, finally balancing the charge of the positive electrode [8].

The working principle of lithium-ion battery is shown in Figure 1.

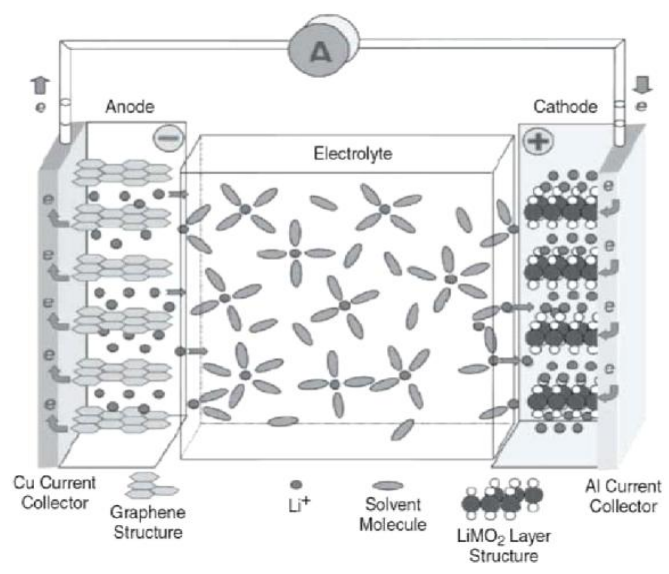

Fig 1:Schematic diagram of working principle of lithium ion battery

ISSN: 0010-8189 
It can be seen from the schematic diagram of the working principle of LiFePO4 battery in the figure above that in order to maintain the charge balance, the same number of lithium ions and electrons are required to participate in the discharge process. And for this process, the migration speed and conductivity of lithium ion and electron also need to be consistent, so the positive and negative electrodes of lithium ion battery must be the mixed conductor of ion and electron.

\subsection{Circuit model of lithium ion battery}

The simplest and most common model of lithium ion battery is usually represented by equivalent circuit model. The equivalent circuit model is a circuit model based on the resistance and capacitance characteristics of the battery during charging and discharging. Typical battery equivalent circuit models are Rint model, Thevenin model and PNGV model, as shown in fig. 2.

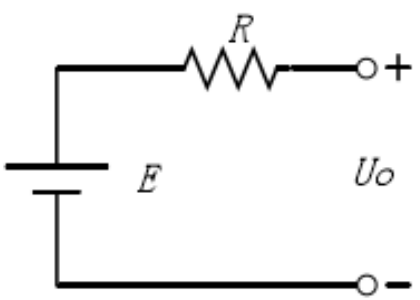

Rint

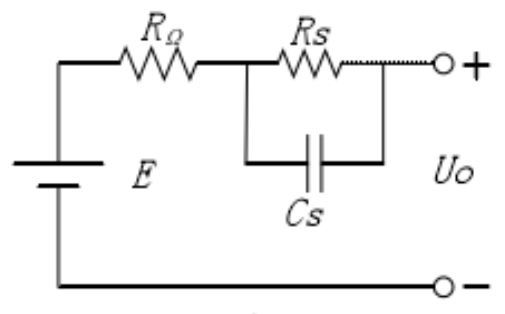

Thevenin

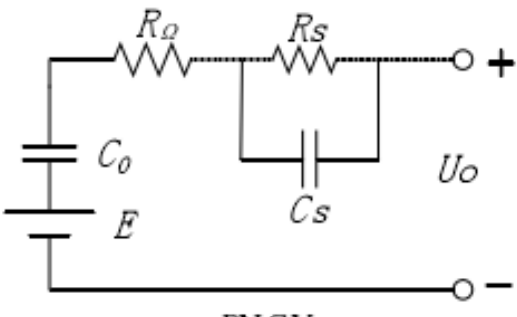

PNGV

Fig 2:Common equivalent circuit models

The Rint model equates the battery to a circuit with a voltage source and a resistor connected in series, which is a simple and ideal model, and does not involve the internal dynamic characteristics of the battery. However, in practice, the internal electromotive force and internal resistance of the battery change due to the influence of the use conditions.

Compared with Rint model, Thevenin model adds RC link to simulate the internal dynamic process of battery. In the model, $\mathrm{R}$ is the ohmic resistance, Rs is the polarization resistance and Cs is the polarization capacitance of the battery. Thevenin model has a simple structure and takes into account the dynamic and static characteristics of the battery, so it is widely used in lithium ion battery modeling.

PNGV model is the standard battery performance model in FreedomCAR Battery Manual Test. In the model, the battery ohmic internal resistance, Rs is the battery polarization internal resistance, Cs is the electric double layer equivalent capacitance, and $\mathrm{C}_{0}$ is the change of open circuit voltage with the accumulation of load current events. The PNGV model considers the characteristics of battery such as polarization and ohmic resistance. The SOC and temperature of battery will affect the change of parameters in the model. Compared with other models, parameter identification is more complex, but it is very difficult to estimate the real-time state of charge, so it is not practical.

\section{Fading mechanism and life influencing factors of lithium batteries}

\subsection{Fading mechanism of lithium ion batteries}

The cycle life of lithium-ion battery is limited. With the continuous use of the battery, its performance will gradually decline. As a rechargeable battery, lithium battery mainly depends on the movement of lithium ions between the positive and negative electrodes. The difference of chemical potential between the two electrodes produces the chemical power of the battery [9-10]. In the process of charging, lithium-ion battery converts electrical energy into chemical energy for storage, while in the process of discharging, chemical energy is converted into electrical energy for load use. In the process of use, with the increase of charge discharge cycles, some irreversible changes will occur in the battery, such as the reduction of electrode active area, the increase of 
current density and polarization; The structure of the active material changes, and the properties of the active particles become worse or even fall off. Corrosion of electrode materials (including collector), decomposition of electrolyte, etc. These irreversible changes will lead to changes in the internal impedance and output current of the battery, which will lead to the attenuation of the battery capacity, and then the cycle life of the lithium-ion battery will be affected.

The main reasons for the decline of anode materials in lithium-ion batteries during recycling are: the formation and growth of SEI film, which makes the contact between anode components worse, which leads to the increase of impedance; The gas produced by embedding solvent into $\mathrm{C}$ electrode leads to the fracture of $\mathrm{C}$ particles; The contact between active material particles becomes worse due to the change of volume in circulation; The precipitated lithium metal reacts with electrolyte to accelerate aging, etc.

As for cathode materials, in the process of recycling, the change of phase change structure of lithium metal oxide, the decomposition of inactive component binder, the oxidation of conductor and the corrosion of current collector will all lead to the increase of internal resistance, the attenuation of capacity and even the generation of gas, which accelerate the aging of lithium ion batteries.

The components of electrolyte in lithium-ion batteries are solvents (commonly used as alkylcarbonate), lithium salts and additives. The process of lithium ion removal and embedding from positive and negative materials interacts with electrolyte from beginning to end. There is a complex redox reaction on this interface. In this process, electrolyte will be lost due to the production of gas or solid products. This is because the gas produced will increase the internal pressure of the battery and lead to the deformation of the battery; The solid products will form passivation film on the surface of the electrode, which will lead to the increase of the polarization of the battery and the decrease of the output voltage. These will have a bad effect on battery capacity and cycle life.

\subsection{Effect of charge discharge ratio on rul}

The cycle charge and discharge process of lithium-ion battery is the use process of the battery. The charge and discharge current, cut-off voltage and charge and discharge mode will affect the life of lithium-ion battery. Figure 3 shows the cycle discharge curves of Li ion battery at different discharge rates.

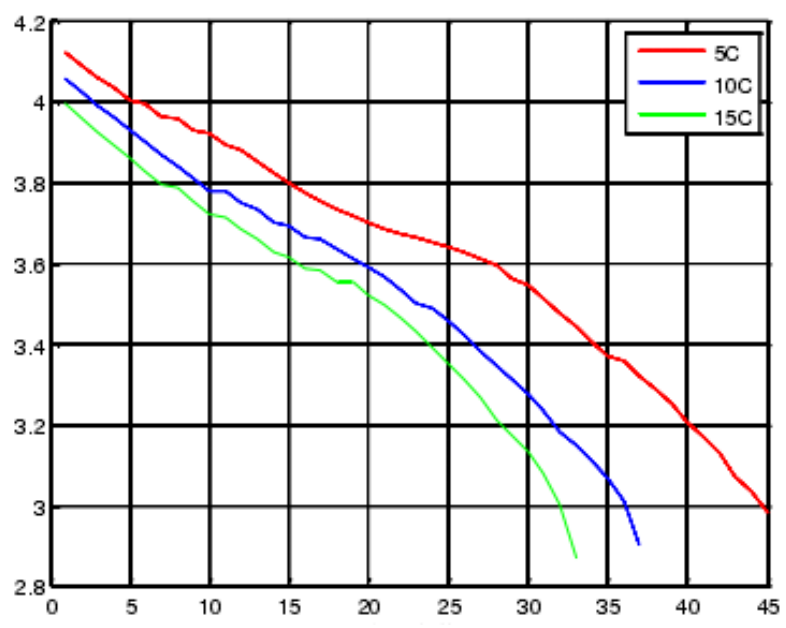

Fig 3:Effect of charge discharge ratio on life of lithium ion battery

In Figure 3, the abscissa represents the number of charge discharge cycles of the lithium-ion battery, and the ordinate represents the capacity of the battery. The dotted line, dotted line, solid line and star line represent the capacity attenuation curves when the discharge rate is $5 \mathrm{C}, 10 \mathrm{C}$ and $15 \mathrm{C}$ respectively. It can be seen from Figure 3 
that the capacity of the battery gradually decreases with the increase of the battery charge discharge cycle, and the larger the discharge rate is, the faster the battery capacity decreases.

The larger the discharge rate of the battery is, the more serious the polarization phenomenon will be. When the diffusion speed of ions is lower than the required speed due to the polarization phenomenon, the capacity of the battery will also be reduced. Through a series of studies, it is concluded that the change of cathode material structure, the increase of SEI film thickness, the decrease of $\mathrm{Li}^{+}$amount and the blockage of diffusion channel are the fundamental reasons for the rapid capacity attenuation. In the case of high current discharge, there is a high demand for the reaction speed of lithium ions, which requires that lithium ions can be quickly embedded and separated from the positive and negative electrodes. Through experimental analysis, Tang Zhiyuan and others believe that: in the process of high current discharge, the battery needs to quickly release a large capacity, which leads to the end of the discharge process before some lithium ions are removed, embedded or passed through the anode material.

\subsection{Life prediction experiment of lithium ion battery}

The capacity of the battery is an important performance index of the battery, and the cycle life reflects the healthy state of the battery. Every charge and discharge of the battery is a cycle. With the recycling of lithium-ion battery, the capacity of the battery will gradually decrease. When the capacity of the battery decays to a certain threshold (usually considered as $80 \%$ of the rated capacity of the battery, but different battery models have different failure thresholds), the battery is considered to be invalid. The residual life prediction diagram of power cell is shown in Figure 4. The remaining service life refers to the remaining service life of the battery after it has been used for a period of time. For example, the cycle life of a power lithium-ion battery is 500 times, which means that the battery can be used for 500 times under normal charge and discharge conditions. If it has been used for 100 times, the remaining service life is 400 times. Generally, the life prediction of lithium-ion battery is divided into physical model method and data-driven method.

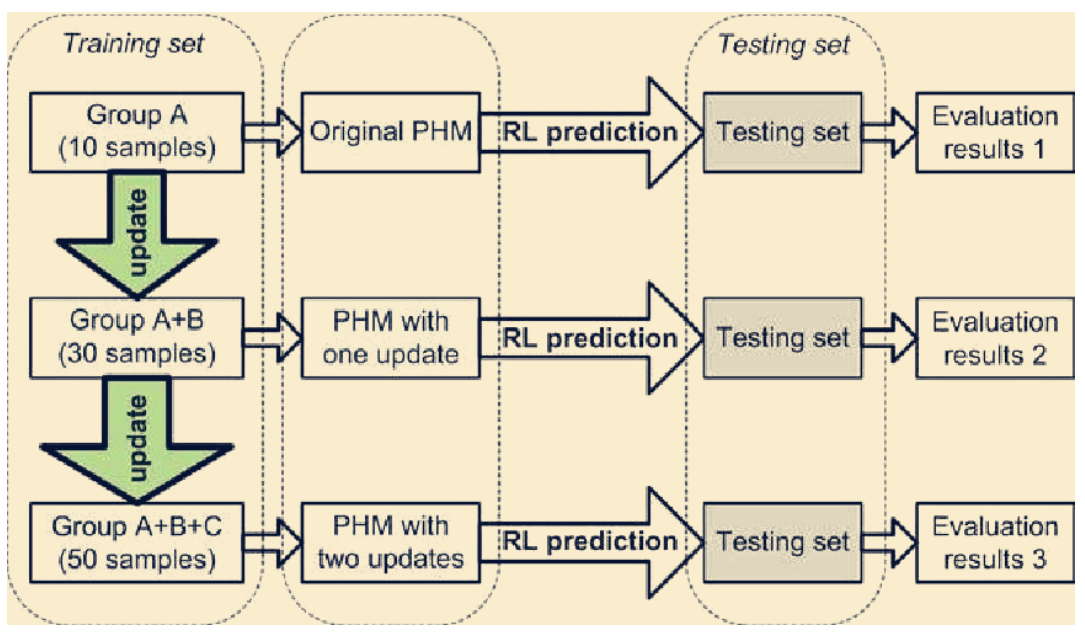

Fig 4:Residual life prediction diagram

Physical model method is based on the study of the internal structure of lithium ion battery, and analyzes the physical and chemical changes in the process of recycling. The formation of SEI film is considered to be the main cause of battery decline. The formation of SEI film consumes lithium ions inside the battery, and the internal resistance between the electrode and electrolyte increases with the thickening of SEI film, which further leads to the decline of battery capacity.

The capacity decay of lithium ion battery reflects the aging of the battery. The capacity refers to the amount of 
charge released in the whole process from the full discharge until the charge is empty. It is usually expressed in ampere hour (Ah), which is expressed as follows:

$$
C=\int_{k}^{k+1} I d t
$$

At constant current, capacity $\mathrm{C}=\mathrm{It}$.

I represents the discharge current of the battery and t represents the discharge time. With the cyclic use of the battery, the capacity of the battery gradually decreases. When the discharged capacity decreases to $80 \%$ of the rated capacity, it is considered that this time is the end of life. For example, if the rated capacity of a certain type of battery is $4 \mathrm{Ah}$, the threshold for marking aging is 3.2Ah. In this paper, the remaining life of the battery is predicted by the experimental data of capacity decay in charge-discharge cycle.

\section{Conventional prediction method of RUL of lithium ion battery}

\subsection{Grey prediction model}

Grey system theory takes small samples and uncertain systems containing both known information and uncertain information as the research object. By generating, developing and extracting valuable information from "part" of known information, it can accurately describe and effectively monitor the system operation behavior and evolution law. $\operatorname{GM}(1,1)$, the model is the core content of grey system theory and the most commonly used grey model. $\operatorname{GM}(1,1)$, firstly, quantifies the known concept of information abstraction in the system, then models the quantized concept, and finally optimizes the model to predict the unknown data. Grey prediction $\operatorname{GM}(1,1)$, the model has been paid attention to by people for a long time.

The prediction results of the remaining service life of lithium-ion batteries using the grey prediction model are shown in Figure 5 below, in which the abscissa is the charge-discharge period and the ordinate is the battery capacity. The adopted data is the latest single cell fatigue test data of a company in Hebei Province.

In fig. 5, the solid line is the actual capacity decline curve of the lithium ion battery, the dotted line is the prediction curve of the remaining life of the lithium ion battery based on the grey prediction model, and the dashed line is the failure threshold. The abscissa of the intersection of the solid line and the dashed line is 114 , which means that when the lithium ion battery is discharged at a current of $2.3 \mathrm{~A}$ at normal temperature for 114 times, the battery charge will reach the failure threshold, which means that the cycle service life of the battery is 114 times.Taking the capacity data of the first 100 cycles of the battery as samples, $\operatorname{GM}(1,1)$ shows that the algorithm can roughly fit the capacity decline curve of the lithium ion battery. The actual cycle life reaches the end of life in the 114th cycle, and the abscissa of the intersection of dotted line and dashed line is 120 , that is, $\operatorname{GM}(1,1)$ is adopted, and the capacity of lithium ion battery predicted by the model decreases to the failure threshold after 120 cycles of charge and discharge, that is, the remaining service life is 20 times. The error between the predicted result and the actual value is 6 cycles. Therefore, the prediction result of grey prediction model accords with the law of capacity attenuation, but the fitting effect is not accurate enough and needs to be improved.

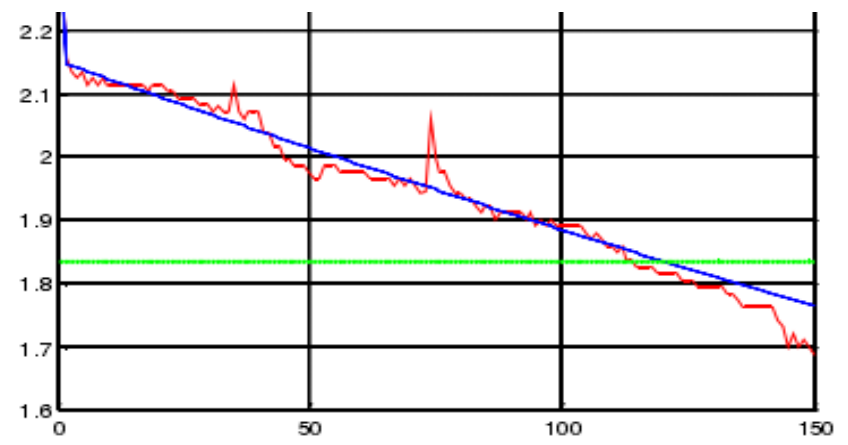

Fig 5:Prediction curve of lithium ion battery life based on grey prediction model 
4.2BP neural network

BP neural network is a kind of multi-layer feedforward neural network. The learning process consists of signal forward propagation and error back propagation. In the process of signal forward propagation, the input signal propagates downward from the input layer through the hidden layer to the output layer. In this process, the neuron state of each layer only affects the neuron state of the next layer; When there is a big difference between the actual output and the expected output in the output layer, it will turn into the back propagation process of error. In this process, the network weights and thresholds will be adjusted according to the prediction error, so that the prediction output of BP neural network will be closer and closer to the expected output, so as to improve the accuracy.

The process of nonlinear function fitting algorithm based on BP neural network can be divided into three steps: BP neural network construction, BP neural network training and BP neural network prediction. The structure of BP neural network is constructed according to the characteristics of fitting nonlinear function. BP neural network is trained with the input and output data of nonlinear function, so that the trained network can predict the output value of nonlinear function.

After 129 iterations to complete the training. In Figure 6, the solid line is the actual capacity decline curve of lithium-ion battery, the dotted line is the prediction curve of residual life of lithium-ion battery based on BP neural network algorithm, and the dotted line is the failure threshold. The abscissa of the intersection of the solid line and the dotted line is 114 , that is to say, the lithium-ion battery is discharged at $2.3 \mathrm{~A}$ current at room temperature. When it is discharged for 114 times, the battery power will reach the failure threshold, that is, the cycle life of the battery is 114 times. The abscissa of the intersection of the dotted line and the dotted line in Figure 6 is 117 , that is, the capacity of the lithium-ion battery predicted by BP neural network algorithm after 117 cycles of charging and discharging is reduced to the failure threshold, so the remaining service life is 17 times. The error between the predicted result and the actual value is three periods. Therefore, BP neural network algorithm is used to predict the remaining service life of lithium-ion battery. Although the fitting curve roughly conforms to the decline trend in the whole process, the predicted decline trend has large fluctuations and poor stability.

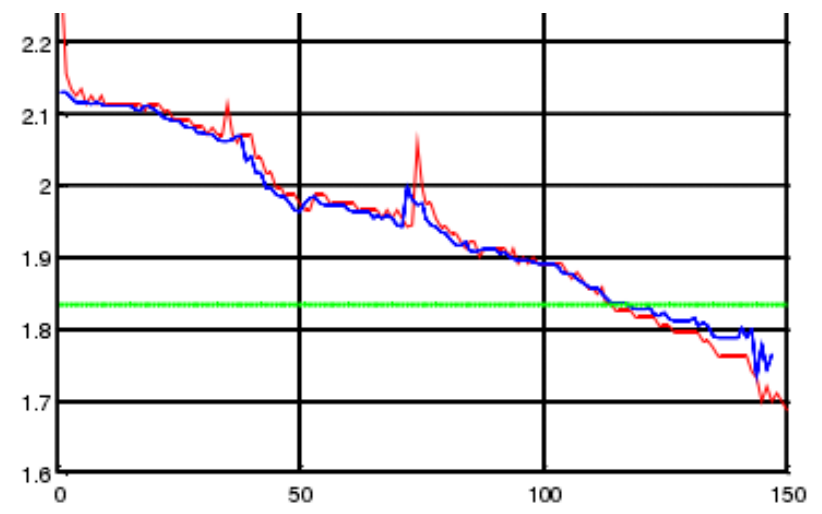

Fig 6:Prediction curve of lithium ion battery life based on BP neural network algorithm

\section{Conclusion}

Lithium ion battery has been considered as the most promising energy storage battery, which can reduce environmental pollution. However, the reliability of lithium-ion batteries has also been the focus of attention, because lithium-ion batteries have low thermal stability and capacity degradation is very serious in high current applications. It is necessary to predict the battery failure in order to reduce the downtime and maintenance costs. Therefore, it is necessary to understand the degradation mechanism of lithium-ion battery and the influence of service factors on its life, and establish a model to predict the remaining service life of lithium-ion battery. 


\section{References}

[1] Ding Yi, Sun Zijian. Research on Temperature Control Performance of Electric Vehicle Power Battery Thermal Management System . Mechanical Manufacturing and Automation, 2020, V.49; No.271 (06): $192-194+202$

[2] Zhou Daohui, Yuan Dewei, Xin Ming. Research on Low Temperature Heating Method for Power Battery of Pure Electric Vehicle . Automotive Electrical Appliances, 2018, 000 (011): 15-17

[3] Zhang Jun, Zhang Lianda, Hu Chunjiao. Research on Heating and Control Strategy of Pure Electric Vehicle Battery Pack . Power Technology, 2016, 40 (009): 1845-1847

[4] Zhang Chengning, Lei Zhiguo, Dong Yugang. Research on Low Temperature Heating Method of Electric Vehicle Lithium Ion Battery . Journal of Beijing University of Technology, 2012 (09): 921-925

[5] Peng Ying, Huang Rui, Yu Xiaoli. Comparative Study on Cooling Schemes of Lithium Ion Power Batteries for Electric Vehicles . Mechanical and Electrical Engineering, 2015, 032 (004): 537-543

[6] Huang Jie. Research on Lithium Battery Management System for Pure Electric Vehicles . Sci Tech Information, 2012, 000 (012): 4-5

[7] Ye Kewei, Xiang Kefeng, Li Liangchao. Simulation Analysis and Research on Heat Generation Characteristics of Vda Square Power Lithium Battery . Power Technology, 2020, V.44; No.355 (04): $45-48+112$

[8] Zhang Pan. Research and Implementation of Thermal Management Technology for Electric Vehicle Power Battery . China's Strategic Emerging Industries, 2018, 000 (030): 113

[9] Zeng Jianhong, Wang Lifang, Liao Chenglin. Optimization Design of Heat Dissipation System for Lithium Ion Power Battery of Electric Vehicle . Acta Electrotechnics Sinica, 2013, 28 (1): 24-29

[10] Liu Xinlei, Cheng Zengmu. Research on Battery Matching Technology of Pure Electric Vehicle . Power Technology, 2018, 042 (003): 367-368376 